\title{
HERTZIAN CONTACT VIBRATIONS UNDER RANDOM EXTERNAL EXCITATION AND SURFACE ROUGHNESS
}

\author{
M. PÄRSSINEN \\ The Marcus Wallenberg Laboratory for Sound and Vibration Research, \\ Department of Vehicle Engineering, KTH, 10044 Stockholm, Sweden
}

(Received 7 February 1997, and in final form 15 July 1997)

\section{INTRODUCTION}

Nayak [1] applied the Fokker-Planck equation [2] to the Hertzian contact problem by considering the excitation to be white noise. Approximate closed form expressions for the standard deviations of displacement and velocity were derived. This procedure was later refined by Hess et al. [3]. By approximating the Hertzian non-linearity, and utilising the stationary solution to the Fokker-Planck equation, a stationary probability density function for the displacement was derived. It was shown numerically that dynamic excitation resulted in a decrease of the mean normal contact compression. By assuming that the adhesion theory of friction is valid, the mean value of the friction force was also found to decrease. The computed results were shown to be in good agreement with measured friction forces at a sliding contact.

The approximations introduced in [3] are in fact not necessary. Utilising exactly the same model as in [3], but without approximating the Hertzian contact stiffness, a new stationary displacement probability density function is derived. By assuming the external and internal excitation to be statistically uncorrelated, a single probability density function represents the combined effect of these dynamic excitations.

Using the results derived herein, the effect of the approximation introduced in [3] is investigated. Furthermore, the results may be used for the evaluation of alternative methods. The accuracy of a perturbation method [4] applied to the Hertzian contact problem is evaluated.

\section{THE MODEL}

The contact is modelled as shown in Figure 1. The surface roughness is represented by the spatially dependent co-ordinate $y_{i}$. Except for the contact region, the rider is modelled as a rigid mass $m$. The compression in the contact region is represented by the non-linear stiffness with coefficient $k$. Using Hertzian contact theory, the relation between an applied static load $P_{0}$ and the resulting compression $\delta$ can be derived [5] as seen in equation (1). For the case of a hemispherical rider sliding over a nominally flat surface, $R$ in (1) is the hemispherical radius. $E$ is the equivalent elastic modulus, which can be derived under the condition that the pressure acting on the first body is equal to the pressure acting on the second.

$$
P_{0}=k \delta^{3 / 2}, \quad k=(4 / 3) \sqrt{R E^{2}} .
$$

Furthermore, the stiffness is zero if the rider loses contact with the rough surface. In Figure 1 , loss of contact corresponds to the inequality $y>y_{i}$. 


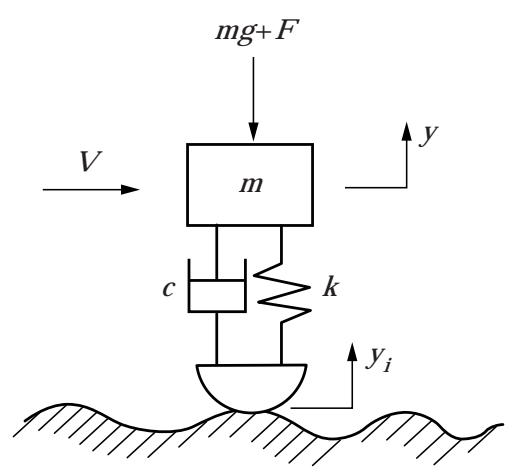

Figure 1. Model of contact between hemispherical rider and rough surface.

It should be noted that, as in [3], the damping $c$ is assumed to be constant at all times, thus unaffected by loss of contact. The governing equation of motion for the rider is then achieved as:

$$
\begin{aligned}
m \ddot{y} & =c\left(\dot{y}_{i}-\dot{y}\right)+k\left(y_{i}-y\right)^{3 / 2}-m g-F, \quad y \leqslant y_{i}, \\
m \ddot{y} & =c\left(\dot{y}_{i}-\dot{y}\right)-m g-F, \quad y>y_{i} .
\end{aligned}
$$

The effects of external dynamic excitation and internal surface roughness excitation are separated in [3]. By assuming that they are statistically uncorrelated, their combined effect may be accounted for by using a single excitation function. Furthermore, the Hertzian non-linear stiffness will not be approximated in this letter. The reason for introducing this approximation in [3] was to remove the static excitation terms. It is noted herein that these static terms may be considered to be part of the non-linear stiffness function. The final equation of motion is thus achieved in a form suitable for application of the stationary solution to the Fokker-Planck equation [2].

The derivation will be discussed briefly. The external force term $F$ is considered to consist of a static and a time-varying term $F=P_{0}+P(t)$. Also introduce variable substitutions. First the displacement $y$ will be referred relative to the static compression $y_{0}$.

$$
y_{\text {rel }}=y-y_{0} \quad y_{0}=\left(\left(P_{0}+m g\right) / k\right)^{2 / 3} .
$$

Furthermore, introduce a change of variables to the dimensionless variables $q, q_{i}$ and $u$, where

$$
q=y_{r e l} / y_{0}, \quad q_{i}=y_{i} / y_{0} ; \quad u=\left(y_{r e l}-y_{i}\right) / y_{0}=q-q_{i} .
$$

Loss of contact now corresponds to the inequality $u>1$.

Equations $(2 \mathrm{a}, \mathrm{b})$ may be grouped into one equation using the Heaviside step function $\mathrm{H}$, which is defined as:

$$
\mathrm{H}(x<0)=0, \quad \mathrm{H}(x \geqslant 0)=1 .
$$

By introducing the substitutions (3-5), the force $F=P_{0}+P(t)$, and the Heaviside function (6) in the equations of motion $(2 a, b)$ the following is achieved.

$$
\ddot{u}+\frac{c}{m} \dot{u}+\left\{-\frac{k[1-H(u-1)]}{m} y_{0}^{1 / 2}(1-u)^{3 / 2}+\frac{1}{m y_{0}}\left(P_{0}+m g\right)\right\}=-\frac{P(t)}{m y_{0}}-\ddot{q}_{i} \equiv F_{3}(t) .
$$


It should be noted that in order to apply the stationary solution of the Fokker-Planck equation to this problem, the static terms $P_{0}$ and $m g$ have here been moved to left side of equation (7). They are thus considered as being a part of the non-linear stiffness. As seen in the right side of equation (7) there are two dynamic excitation terms. The first term includes $P(t)$, which is a white noise external excitation with zero mean value level and a constant one-sided spectral density $W_{0}$. The one-sided spectral density for the term $P(t) / m y_{0}$ is thus $Z_{1}=W_{0} / m^{2} y_{0}^{2}$. The second term is the second time derivative of the non-dimensionalised surface roughness $q_{i}$. The same statistical properties as in [3] are assumed. Thus the term $\ddot{q}_{i}$ is a white noise process with zero mean value and a constant one-sided spectral density $Z_{2}=4 L V^{3} / y_{0}^{2}$. $L$ is a constant to be fitted to surface roughness measurements, and $V$ is the horizontal velocity of the rider.

By assuming that the external excitation and the internal surface roughness excitation are statistically uncorrelated, the statistical properties of $F_{3}(t)$ in equation (7) can be derived trivially. $F_{3}(t)$ is simply a white noise process with zero mean value level and a constant one-sided spectral density $Z_{3}$, given by equation (8).

$$
Z_{3}=Z_{1}+Z_{2}=\left(1 / y_{0}^{2}\right)\left(W_{0} / m^{2}+4 L V^{3}\right) .
$$

\section{THE STATIONARY SOLUTION}

The Fokker-Planck equation [2] is an expression for the evolution in time of the second order probability density function for Markoff processes. If the conditional probability tends to a limiting stationary probability density function, the stationary form of the Fokker-Planck equation is achieved. By applying the stationary form of the FokkerPlanck equation to the single-degree-of-freedom system equation (9), with the non-linear stiffness $g(u)$, it is possible to show [2] that the stationary probability density function $p_{s}(u)$ for the displacement is achieved as equation (10). This is for the case in which the excitation $F_{3}(t)$ is a white noise signal with a constant one-sided spectral density $Z_{3}$.

$$
\ddot{u}+\beta \dot{u}+g(u)=F_{3}(t), \quad p_{s}(u)=C \exp \left\{-\frac{4 \beta}{Z_{3}} \int_{0}^{u} g\left(u_{1}\right) \mathrm{d} u_{1}\right\}
$$

From the probability density function (10), statistical moments of the stationary level of displacement can be computed through integration. The equation of motion (7) is now identified with equation (9). By performing the integration (10) and using the statistical properties (8) of the dynamic excitation, a closed form for the displacement probability density function is achieved as (11).

$p_{s}(u)=C \exp \left\{-\frac{4 c y_{0}^{2}}{\left(W_{0}+4 m^{2} L V^{3}\right)}\left[\frac{2 k y_{0}^{1 / 2}}{5}\left[(1-H(u-1))(1-u)^{5 / 2}-1\right]+\left(P_{0}+m g\right) \frac{u}{y_{0}}\right]\right\}$.

As previously mentioned, the derived probability density function differs from the one derived in [3] in the sense that no approximation has been introduced for the Hertzian non-linearity. Furthermore, by assuming that the external excitation and the surface roughness are statistically uncorrelated, a single probability density function incorporates the combined effect of these two sources of vibration. The cases of sole external- or sole surface roughness excitation are achieved by letting either $L$ or $W_{0}$ be zero. 
TABLE 1

Base set of parameters

\begin{tabular}{cccccc}
\hline$L\left(\mathrm{~m}^{-1}\right)$ & $V(\mathrm{~m} / \mathrm{s})$ & $m(\mathrm{~kg})$ & $\zeta$ & $R(\mathrm{~m})$ & $P_{0}(\mathrm{~N})$ \\
\hline 0.5 & 0.1 & 0.5 & 0.01 & 0.01 & 5 \\
\hline
\end{tabular}

\section{NUMERICAL RESULTS}

By utilising the derived probability density function (11), mean value levels of the dimensionless relative displacement $u$ can be computed through numerical integration. The computed results will be compared with the results in [3]. As those results were derived by approximating the Hertzian non-linear stiffness, they will be denoted as $\langle u\rangle_{a p p r}$. Furthermore, a comparison will be made to results which were derived by using a perturbation method [4]. Those results which will be denoted as $\langle u\rangle_{\text {perturb }}$. The same parameters as used in [3] and [4] will be used for easy comparison. The case of sole surface roughness excitation will be investigated, with the base set of parameters given in Table 1. The result of the parameter study is presented in Table 2 .

It should be noted that the damping in Table 1 is given by the dimensionless damping ratio $\zeta$, which is defined by

$$
2 \omega_{0} \zeta=c / m .
$$

The factor $\omega_{0}$ is the small amplitude natural frequency, which is derived by linearising equation (7) under the assumption that $u \ll 1$. A relation between the viscous damping $c$ and the damping ratio $\zeta$ is thus achieved.

$$
c=\zeta \sqrt{6 m k y_{0}^{1 / 2}} .
$$

TABLE 2

\begin{tabular}{|c|c|c|c|c|c|c|c|}
\hline Case & Parar & neters & $\langle u\rangle$ & $\langle u\rangle_{a p p r}$ & $\langle u\rangle_{\text {perturb }}$ & $\left(\langle u\rangle_{a p p r}-\langle u\rangle\right) /\langle u\rangle$ & $\left(\langle u\rangle_{\text {perturb }}-\langle u\rangle\right) /\langle u\rangle$ \\
\hline 1 & Base $†$ & & $0 \cdot 0573$ & $0 \cdot 0584$ & 0.0507 & 1.9 & $-11 \cdot 5$ \\
\hline 2 & $m(\mathrm{~kg})$ & $1 \cdot 0$ & $0 \cdot 0871$ & $0 \cdot 0889$ & 0.0703 & $2 \cdot 1$ & $-19 \cdot 3$ \\
\hline 3 & & $0 \cdot 8$ & $0 \cdot 0233$ & $0 \cdot 0245$ & 0.0235 & $5 \cdot 2$ & 0.9 \\
\hline 4 & $\zeta$ & 0.0075 & 0.0853 & $0 \cdot 0871$ & 0.0692 & $2 \cdot 1$ & $-18 \cdot 9$ \\
\hline 5 & & $0 \cdot 0200$ & $0 \cdot 0243$ & 0.0255 & 0.0244 & 4.9 & 0.4 \\
\hline 6 & $R(\mathrm{~m})$ & $0 \cdot 0500$ & $0 \cdot 0830$ & $0 \cdot 0847$ & $0 \cdot 0677$ & $2 \cdot 1$ & $-18 \cdot 4$ \\
\hline 7 & & $0 \cdot 0001$ & $0 \cdot 0223$ & $0 \cdot 0235$ & $0 \cdot 0226$ & $5 \cdot 4$ & $1 \cdot 4$ \\
\hline 8 & $P_{0}(\mathrm{~N})$ & $3 \cdot 5$ & 0.0870 & $0 \cdot 0888$ & $0 \cdot 0702$ & $2 \cdot 1$ & $-19 \cdot 3$ \\
\hline 9 & & $10 \cdot 0$ & $0 \cdot 0228$ & 0.0240 & 0.0230 & $5 \cdot 3$ & 0.9 \\
\hline 10 & $L\left(\mathrm{~m}^{-1}\right)$ & 0.65 & $0 \cdot 0823$ & $0 \cdot 0840$ & 0.0673 & $2 \cdot 1$ & $-18 \cdot 2$ \\
\hline 11 & & $0 \cdot 25$ & $0 \cdot 0243$ & 0.0255 & $0 \cdot 0244$ & 4.9 & $0 \cdot 4$ \\
\hline 12 & $V(\mathrm{~m} / \mathrm{s})$ & $0 \cdot 11$ & $0 \cdot 0851$ & 0.0869 & 0.0691 & $2 \cdot 1$ & $-18 \cdot 8$ \\
\hline 13 & & 0.08 & $0 \cdot 0249$ & $0 \cdot 0261$ & 0.0250 & $4 \cdot 8$ & $0 \cdot 4$ \\
\hline
\end{tabular}

Mean value levels under internal surface roughness excitation

$\dagger$ Base refers to Table 1. 


\section{SUMMARY AND CONCLUSIONS}

By applying the stationary solution of the Fokker-Planck equation to the Hertzian contact problem, the resulting stationary probability density function for the relative displacement has been derived. The exact Hertzian stiffness is used in this letter. For the derivation presented here, it was furthermore assumed that the external random excitation and the internal surface roughness excitation are statistically uncorrelated. Under this assumption a single probability density function sufficiently represents the combined effects of the two excitation mechanisms. Computed statistical moments, using the exact Hertzian non-linear contact stiffness, have been compared with the previous approximate Fokker-Planck solution [3]. The approximation introduced in [3] tends to overestimate the mean relative displacement, but the error is usually less than about five percent. On the other hand, the perturbation method [4] tends to underestimate the mean relative displacement, and the error can reach up to about twenty percent.

For further reference, the results presented herein should provide updated numerical values for resulting statistical moments of the mean vibratory separation.

\section{ACKNOWLEDGMENT}

The funding provided by Scania is gratefully acknowledged.

\section{REFERENCES}

1. P. R. NAYAK 1972 Journal of Sound and Vibration 22, 297-322. Contact vibrations.

2. T. K. Caughey 1963 Journal of the Acoustical Society of America 35, 1683-1692. Derivation and application of the Fokker-Planck equation to discrete nonlinear dynamic systems subjected to white random excitation.

3. D. P. Hess, A. Soom and C. H. Kim 1992 Journal of Sound and Vibration 153, 491-508. Normal vibrations and friction at a Hertzian contact under random excitation: theory and experiments.

4. D. P. Hess and A. Soom 1993 Journal of Sound and Vibration 164, 317-326. Normal vibrations and friction at a Hertzian contact under random excitation: perturbation solution.

5. K. L. Johnson 1985 Contact mechanics. Cambridge: Cambridge University Press. 